\title{
Neutrino Telescopes as a Probe of Broken $\mu-\tau$ Symmetry
}

\author{
Zhi-zhong Xing * \\ Institute of High Energy Physics, Chinese Academy of Sciences, \\ P.O. Box 918, Beijing 100049, China
}

\begin{abstract}
It is known that neutrino oscillations may map $\phi_{e}: \phi_{\mu}: \phi_{\tau}=1: 2: 0$, the initial flavor ratios of ultrahigh-energy neutrino fluxes produced from a distant astrophysical source, into $\phi_{e}^{\mathrm{D}}: \phi_{\mu}^{\mathrm{D}}: \phi_{\tau}^{\mathrm{D}}=1: 1: 1$ at the detector of a neutrino telescope. We remark that this naive expectation is only valid in the $\mu-\tau$ symmetry limit, in which two neutrino mixing angles satisfy $\theta_{13}=0$ and $\theta_{23}=\pi / 4$. Allowing for the slight breaking of $\mu-\tau$ symmetry, we find $\phi_{e}^{\mathrm{D}}: \phi_{\mu}^{\mathrm{D}}: \phi_{\tau}^{\mathrm{D}}=(1-2 \Delta):(1+\Delta):(1+\Delta)$ with $\Delta$ characterizing the combined effect of $\theta_{13} \neq 0$ and $\theta_{23} \neq \pi / 4$. Current neutrino oscillation data indicate $-0.1 \lesssim \Delta \lesssim+0.1$. We also look at the possibility to probe $\Delta$ by detecting the $\bar{\nu}_{e}$ flux of $E_{\bar{\nu}_{e}} \approx 6.3 \mathrm{PeV}$ via the Glashow resonance channel $\bar{\nu}_{e} e \rightarrow W^{-} \rightarrow$ anything.
\end{abstract}

PACS number(s): 14.60.Lm, 14.60.Pq, 95.85.Ry

*E-mail: xingzz@mail.ihep.ac.cn 
1 The solar [1], atmospheric [2], reactor [3] and accelerator [4] neutrino experiments have convinced us of the existence of neutrino oscillations and opened a new window to physics beyond the standard model. Given the basis in which the flavor eigenstates of charged leptons are identified with their mass eigenstates, the phenomenon of neutrino mixing can simply be described by a $3 \times 3$ unitary matrix $V$ which links the neutrino flavor eigenstates $\left(\nu_{e}, \nu_{\mu}, \nu_{\tau}\right)$ to the neutrino mass eigenstates $\left(\nu_{1}, \nu_{2}, \nu_{3}\right)$ :

$$
\left(\begin{array}{c}
\nu_{e} \\
\nu_{\mu} \\
\nu_{\tau}
\end{array}\right)=\left(\begin{array}{ccc}
V_{e 1} & V_{e 2} & V_{e 3} \\
V_{\mu 1} & V_{\mu 2} & V_{\mu 3} \\
V_{\tau 1} & V_{\tau 2} & V_{\tau 3}
\end{array}\right)\left(\begin{array}{c}
\nu_{1} \\
\nu_{2} \\
\nu_{3}
\end{array}\right) .
$$

In the so-called standard parametrization of $V[5], V_{e 2}=\sin \theta_{12} \cos \theta_{13}, V_{e 3}=\sin \theta_{13} e^{-i \delta}$ and $V_{\mu 3}=\sin \theta_{23} \cos \theta_{13}$. Here we have omitted the Majorana CP-violating phases from $V$, because they are irrelevant to the properties of neutrino oscillations to be discussed. A global analysis of current experimental data (see, e.g., Ref. [6]) points to $\theta_{13}=0$ and $\theta_{23}=\pi / 4$, which motivate a number of authors to consider the $\mu-\tau$ permutation symmetry for model building [7]. In the limit of $\mu-\tau$ symmetry, the (effective) neutrino mass matrix $M_{\nu}$ takes the form

$$
M_{\nu}=V_{0} \bar{M}_{\nu} V_{0}^{T}=\left(\begin{array}{lll}
a & b & b \\
b & c & d \\
b & d & c
\end{array}\right)
$$

where $\bar{M}_{\nu} \equiv \operatorname{Diag}\left\{m_{1}, m_{2}, m_{3}\right\}$ with $m_{i}$ (for $i=1,2,3$ ) being three neutrino masses, and $V_{0}$ is a special pattern of $V$ with $\theta_{13}=0$ and $\theta_{23}=\pi / 4$. Note that $\theta_{12}$ is arbitrary and $\delta$ is not well-defined in $V_{0}$. The parameters $a, b, c$ and $d$ of $M_{\nu}$ are in general complex and can produce the desired Majorana CP-violating phases for $V_{0}$. Any slight breaking of $\mu-\tau$ symmetry in $M_{\nu}$ will result in non-vanishing $\theta_{13}$ and small departure of $\theta_{23}$ from $\pi / 4$. Nontrivial $\delta$ can also be generated from the breaking of $\mu-\tau$ symmetry, leading to the effect of $\mathrm{CP}$ violation in neutrino oscillations.

The main purpose of this paper is to investigate how the effect of $\mu-\tau$ symmetry breaking can show up at a neutrino telescope. We expect that IceCube [8] and other second-generation neutrino telescopes [9] are able to detect the fluxes of ultrahigh-energy $\nu_{e}\left(\bar{\nu}_{e}\right), \nu_{\mu}\left(\bar{\nu}_{\mu}\right)$ and $\nu_{\tau}\left(\bar{\nu}_{\tau}\right)$ neutrinos produced from very distant astrophysical sources. If the initial neutrino fluxes are produced via the decay of pions created from $p p$ and (or) $p \gamma$ collisions, their flavor content can be written as

$$
\left\{\phi_{e}, \phi_{\mu}, \phi_{\tau}\right\}=\left\{\frac{1}{3}, \frac{2}{3}, 0\right\} \phi_{0}
$$

where $\phi_{\alpha}$ (for $\left.\alpha=e, \mu, \tau\right)$ denotes the sum of $\nu_{\alpha}$ and $\bar{\nu}_{\alpha}$ fluxes, and $\phi_{0}=\phi_{e}+\phi_{\mu}+\phi_{\tau}$ is the total flux of neutrinos and antineutrinos of all flavors. Due to neutrino oscillations, the flavor composition of such cosmic neutrino fluxes to be measured at the detector of a neutrino telescope has been expected to be

$$
\left\{\phi_{e}^{\mathrm{D}}, \phi_{\mu}^{\mathrm{D}}, \phi_{\tau}^{\mathrm{D}}\right\}=\left\{\frac{1}{3}, \frac{1}{3}, \frac{1}{3}\right\} \phi_{0} .
$$


However, it is worth remarking that this naive expectation is only true in the limit of $\mu-\tau$ symmetry (or equivalently, $\theta_{13}=0$ and $\theta_{23}=\pi / 4$ ). Allowing for the slight breaking of $\mu-\tau$ symmetry, we are going to show that

$$
\left\{\phi_{e}^{\mathrm{D}}: \phi_{\mu}^{\mathrm{D}}: \phi_{\tau}^{\mathrm{D}}\right\}=\{(1-2 \Delta):(1+\Delta):(1+\Delta)\}
$$

holds to an excellent degree of accuracy, where $\Delta$ characterizes the effect of $\mu-\tau$ symmetry breaking (i.e., the combined effect of $\theta_{13} \neq 0$ and $\theta_{23} \neq \pi / 4$ ). We obtain $-0.1 \lesssim \Delta \lesssim+0.1$ from current neutrino oscillation data. We find that it is also possible to probe $\Delta$ by detecting the $\bar{\nu}_{e}$ flux of $E_{\bar{\nu}_{e}} \approx 6.3 \mathrm{PeV}$ via the well-known Glashow resonance (GR) channel $\bar{\nu}_{e} e \rightarrow W^{-} \rightarrow$ anything [10] at a neutrino telescope.

2 Let us define $\phi_{\alpha}^{(\mathrm{D})} \equiv \phi_{\nu_{\alpha}}^{(\mathrm{D})}+\phi_{\bar{\nu}_{\alpha}}^{(\mathrm{D})}($ for $\alpha=e, \mu, \tau)$ throughout this paper, where $\phi_{\nu_{\alpha}}^{(\mathrm{D})}$ and $\phi_{\bar{\nu}_{\alpha}}^{(\mathrm{D})}$ denote the $\nu_{\alpha}$ and $\bar{\nu}_{\alpha}$ fluxes, respectively. As for the ultrahigh-energy neutrino fluxes produced from the pion-muon decay chain with $\phi_{\nu_{\tau}}=\phi_{\bar{\nu}_{\tau}}=0$, the relationship between $\phi_{\nu_{\alpha}}$ (or $\phi_{\bar{\nu}_{\alpha}}$ ) and $\phi_{\nu_{\alpha}}^{\mathrm{D}}\left(\right.$ or $\left.\phi_{\bar{\nu}_{\alpha}}^{\mathrm{D}}\right)$ is given by $\phi_{\nu_{\alpha}}^{\mathrm{D}}=\phi_{\nu_{e}} P_{e \alpha}+\phi_{\nu_{\mu}} P_{\mu \alpha}$ or $\phi_{\bar{\nu}_{\alpha}}^{\mathrm{D}}=\phi_{\bar{\nu}_{e}} \bar{P}_{e \alpha}+\phi_{\bar{\nu}_{\mu}} \bar{P}_{\mu \alpha}$, in which $P_{\beta \alpha}$ and $\bar{P}_{\beta \alpha}$ (for $\alpha=e, \mu, \tau$ and $\beta=e$ or $\mu$ ) stand respectively for the oscillation probabilities $P\left(\nu_{\beta} \rightarrow \nu_{\alpha}\right)$ and $P\left(\bar{\nu}_{\beta} \rightarrow \bar{\nu}_{\alpha}\right)$. Because the Galactic distances far exceed the observed neutrino oscillation lengths, $P_{\beta \alpha}$ and $\bar{P}_{\beta \alpha}$ are actually averaged over many oscillations. Then we obtain $\bar{P}_{\beta \alpha}=P_{\beta \alpha}$ and

$$
P_{\beta \alpha}=\sum_{i=1}^{3}\left|V_{\alpha i}\right|^{2}\left|V_{\beta i}\right|^{2},
$$

where $V_{\alpha i}$ and $V_{\beta i}$ (for $\alpha, \beta=e, \mu, \tau$ and $i=1,2,3$ ) denote the matrix elements of $V$ defined in Eq. (1). The relationship between $\phi_{\alpha}$ and $\phi_{\alpha}^{\mathrm{D}}$ turns out to be

$$
\phi_{\alpha}^{\mathrm{D}}=\phi_{e} P_{e \alpha}+\phi_{\mu} P_{\mu \alpha} .
$$

To be explicit, we have

$$
\begin{aligned}
& \phi_{e}^{\mathrm{D}}=\frac{\phi_{0}}{3}\left(P_{e e}+2 P_{\mu e}\right), \\
& \phi_{\mu}^{\mathrm{D}}=\frac{\phi_{0}}{3}\left(P_{e \mu}+2 P_{\mu \mu}\right), \\
& \phi_{\tau}^{\mathrm{D}}=\frac{\phi_{0}}{3}\left(P_{e \tau}+2 P_{\mu \tau}\right) .
\end{aligned}
$$

It is then possible to evaluate the relative magnitudes of $\phi_{e}^{\mathrm{D}}, \phi_{\mu}^{\mathrm{D}}$ and $\phi_{\tau}^{\mathrm{D}}$ by using Eqs. (1), (6) and (8).

In order to clearly show the effect of $\mu-\tau$ symmetry breaking on the neutrino fluxes to be detected at neutrino telescopes, we define two small quantities $(0<\epsilon \ll 1$ and $|\varepsilon| \ll 1)$ :

$$
\epsilon \equiv \theta_{13}, \quad \varepsilon \equiv \theta_{23}-\frac{\pi}{4} .
$$

In other words, $\epsilon$ and $\varepsilon$ measure the deviation of $\theta_{13}$ from zero and the departure of $\theta_{23}$ from $\pi / 4$, respectively. Then $\left|V_{\alpha i}\right|^{2}$ (for $\alpha=e, \mu, \tau$ and $i=1,2,3$ ) can be expressed as 


$$
\begin{aligned}
{\left[\begin{array}{ccc}
\left|V_{e 1}\right|^{2} & \left|V_{e 2}\right|^{2} & \left|V_{e 3}\right|^{2} \\
\left|V_{\mu 1}\right|^{2} & \left|V_{\mu 2}\right|^{2} & \left|V_{\mu 3}\right|^{2} \\
\left|V_{\tau 1}\right|^{2} & \left|V_{\tau 2}\right|^{2} & \left|V_{\tau 3}\right|^{2}
\end{array}\right]=} & \frac{1}{2}\left[\begin{array}{ccc}
2 \cos ^{2} \theta_{12} & 2 \sin ^{2} \theta_{12} & 0 \\
\sin ^{2} \theta_{12} & \cos ^{2} \theta_{12} & 1 \\
\sin ^{2} \theta_{12} & \cos ^{2} \theta_{12} & 1
\end{array}\right]+\varepsilon\left[\begin{array}{ccc}
0 & 0 & 0 \\
-\sin ^{2} \theta_{12} & -\cos ^{2} \theta_{12} & 1 \\
\sin ^{2} \theta_{12} & \cos ^{2} \theta_{12} & -1
\end{array}\right] \\
& +\frac{\epsilon}{2} \sin 2 \theta_{12} \cos \delta\left[\begin{array}{ccc}
0 & 0 & 0 \\
1 & -1 & 0 \\
-1 & 1 & 0
\end{array}\right]+\mathcal{O}\left(\varepsilon^{2}\right)+\mathcal{O}\left(\epsilon^{2}\right) .
\end{aligned}
$$

Combining Eqs. (6) and (10) allows us to calculate $P_{\beta \alpha}$. After a straightforward calculation, we obtain

$$
\begin{aligned}
& P_{e e}+2 P_{\mu e}=1-\varepsilon \sin ^{2} 2 \theta_{12}+\frac{\epsilon}{2} \sin 4 \theta_{12} \cos \delta+\mathcal{O}\left(\varepsilon^{2}\right)+\mathcal{O}\left(\epsilon^{2}\right) \\
& P_{e \mu}+2 P_{\mu \mu}=1+\frac{\varepsilon}{2} \sin ^{2} 2 \theta_{12}-\frac{\epsilon}{4} \sin 4 \theta_{12} \cos \delta+\mathcal{O}\left(\varepsilon^{2}\right)+\mathcal{O}\left(\epsilon^{2}\right) \\
& P_{e \tau}+2 P_{\mu \tau}=1+\frac{\varepsilon}{2} \sin ^{2} 2 \theta_{12}-\frac{\epsilon}{4} \sin 4 \theta_{12} \cos \delta+\mathcal{O}\left(\varepsilon^{2}\right)+\mathcal{O}\left(\epsilon^{2}\right)
\end{aligned}
$$

Substituting Eq. (11) into Eq. (8), we arrive at

$$
\begin{aligned}
\phi_{e}^{\mathrm{D}} & =\frac{\phi_{0}}{3}(1-2 \Delta), \\
\phi_{\mu}^{\mathrm{D}} & =\frac{\phi_{0}}{3}(1+\Delta), \\
\phi_{\tau}^{\mathrm{D}} & =\frac{\phi_{0}}{3}(1+\Delta),
\end{aligned}
$$

where

$$
\Delta=\frac{1}{4}\left(2 \varepsilon \sin ^{2} 2 \theta_{12}-\epsilon \sin 4 \theta_{12} \cos \delta\right)+\mathcal{O}\left(\varepsilon^{2}\right)+\mathcal{O}\left(\epsilon^{2}\right) .
$$

Eq. (5) is therefore proved by Eq. (12). One can see that $\phi_{e}^{\mathrm{D}}+\phi_{\mu}^{\mathrm{D}}+\phi_{\tau}^{\mathrm{D}}=\phi_{0}$ holds. Some discussions are in order.

(1) The small parameter $\Delta$ characterizes the overall effect of $\mu-\tau$ symmetry breaking. Allowing $\delta$ to vary between 0 and $\pi$, we may easily obtain the lower and upper bounds of $\Delta$ for given values of $\theta_{12}(<\pi / 4), \epsilon$ and $\varepsilon$ : $-\Delta_{\text {bound }} \leq \Delta \leq+\Delta_{\text {bound }}$, where

$$
\Delta_{\text {bound }}=\frac{1}{4}\left(2|\varepsilon| \sin ^{2} 2 \theta_{12}+\epsilon \sin 4 \theta_{12}\right)+\mathcal{O}\left(\varepsilon^{2}\right)+\mathcal{O}\left(\epsilon^{2}\right) .
$$

It is obvious that $\Delta=-\Delta_{\text {bound }}$ when $\varepsilon<0$ and $\delta=0$, and $\Delta=+\Delta_{\text {bound }}$ when $\varepsilon>0$ and $\delta=\pi$. A global analysis of current neutrino oscillation data [6] indicates $30^{\circ}<\theta_{12}<38^{\circ}$, $\epsilon<10^{\circ}(\approx 0.17)$ and $|\varepsilon|<9^{\circ}(\approx 0.16)$ at the $99 \%$ confidence level, but the CP-violating phase $\delta$ is entirely unrestricted. Using these constraints, we analyze the allowed range of $\Delta$ and its dependence on $\delta$. The maximal value of $\Delta_{\text {bound }}$ (i.e., $\Delta_{\text {bound }} \approx 0.098$ ) appears when $|\varepsilon|$ and $\epsilon$ approach their respective upper limits and $\theta_{12} \approx 33^{\circ}$ holds, as one can clearly see from Fig. $1(\mathrm{~A})$. Indeed, we find that $\Delta_{\text {bound }}$ is not very sensitive to the variation of $\theta_{12}$ in its allowed region.

Provided $\epsilon=0$ (i.e., $\theta_{13}=0$ ) holds, we easily obtain $\Delta_{\text {bound }}=0.5|\varepsilon| \sin ^{2} 2 \theta_{12}<0.074$ when $\theta_{12}$ approaches its upper limit. If $\varepsilon=0$ (i.e., $\theta_{23}=\pi / 4$ ) holds, nevertheless, we find 
$\Delta_{\text {bound }}=0.25 \epsilon \sin 4 \theta_{12}<0.038$ as $\theta_{12}$ approaches its lower limit. We observe that $\Delta_{\text {bound }}$ is more sensitive to the deviation of $\theta_{23}$ from $\pi / 4$.

(2) Of course, $\Delta=0$ exactly holds when $\epsilon=\varepsilon=0$ is taken. Because the sign of $\varepsilon$ and the range of $\delta$ are both unknown, we are now unable to rule out the nontrivial possibility $\Delta \approx 0$ in the presence of $\epsilon \neq 0$ and $\varepsilon \neq 0$. In other words, $\Delta$ may be vanishing or extremely small if its two leading terms cancel each other. It is straightforward to arrive at $\Delta \approx 0$ from Eq. (13), if the condition

$$
\frac{\varepsilon}{\epsilon}=\cot 2 \theta_{12} \cos \delta
$$

is satisfied. Due to $|\cos \delta| \leq 1$, Eq. (15) imposes a strong constraint on the magnitude of $\varepsilon / \epsilon$. The dependence of $\varepsilon / \epsilon$ on $\delta$ is illustrated in Fig. 1(B), where $\theta_{12}$ varies in its allowed range. One can see that $|\varepsilon| / \epsilon<0.6$ is necessary to hold, such that a large cancellation between two leading terms of $\Delta$ is possible to take place.

The implication of Fig. 1 on high-energy neutrino telescopes is two-fold. On the one hand, an observable signal of $\Delta \neq 0$ at a neutrino telescope implies the existence of significant $\mu-\tau$ symmetry breaking. If a signal of $\Delta \neq 0$ does not show up at a neutrino telescope, on the other hand, one cannot conclude that the $\mu-\tau$ symmetry is an exact or almost exact symmetry. It is therefore meaningful to consider the complementarity between neutrino telescopes and terrestrial neutrino oscillation experiments [11], in order to finally pin down the parameters of neutrino mixing and leptonic CP violation.

(3) To illustrate, we define three neutrino flux ratios

$$
\begin{aligned}
R_{e} & \equiv \frac{\phi_{e}^{\mathrm{D}}}{\phi_{\mu}^{\mathrm{D}}+\phi_{\tau}^{\mathrm{D}}}, \\
R_{\mu} & \equiv \frac{\phi_{\mu}^{\mathrm{D}}}{\phi_{\tau}^{\mathrm{D}}+\phi_{e}^{\mathrm{D}}}, \\
R_{\tau} & \equiv \frac{\phi_{\tau}^{\mathrm{D}}}{\phi_{e}^{\mathrm{D}}+\phi_{\mu}^{\mathrm{D}}},
\end{aligned}
$$

which may serve as the working observables at neutrino telescopes [12]. At least, $R_{\mu}$ can be extracted from the ratio of muon tracks to showers at IceCube [8], even if those electron and tau events cannot be disentangled. Taking account of Eq. (12), we approximately obtain

$$
\begin{aligned}
R_{e} & \approx \frac{1}{2}-\frac{3}{2} \Delta, \\
R_{\mu} & \approx \frac{1}{2}+\frac{3}{4} \Delta, \\
R_{\tau} & \approx \frac{1}{2}+\frac{3}{4} \Delta .
\end{aligned}
$$

It turns out that $R_{e}$ is most sensitive to the effect of $\mu-\tau$ symmetry breaking.

As a straightforward consequence of $\phi_{\mu}^{\mathrm{D}}=\phi_{\tau}^{\mathrm{D}}$ shown in Eq. (12), $R_{\mu}=R_{\tau}$ holds no matter whether $\Delta$ vanishes or not. This interesting observation implies that the " $\mu-\tau$ " symmetry between $R_{\mu}$ and $R_{\tau}$ is actually insensitive to the breaking of $\mu-\tau$ symmetry in the 
neutrino mass matrix $M_{\nu}$. If both $R_{e}$ and $R_{\mu}$ are measured at a neutrino telescope, one can then extract the information about $\Delta$ from the difference of these two observables:

$$
R_{\mu}-R_{e}=\frac{9}{4} \Delta
$$

Taking $\Delta=\Delta_{\text {bound }} \approx 0.1$, we get $R_{\mu}-R_{e} \lesssim 0.22$.

3 We proceed to discuss the possibility to probe the breaking of $\mu-\tau$ symmetry by detecting the $\bar{\nu}_{e}$ flux from distant astrophysical sources through the Glashow resonance (GR) channel $\bar{\nu}_{e} e \rightarrow W^{-} \rightarrow$ anything [10]. The latter can take place over a narrow energy interval around the $\bar{\nu}_{e}$ energy $E_{\bar{\nu}_{e}}^{\mathrm{GR}} \approx M_{W}^{2} / 2 m_{e} \approx 6.3 \mathrm{PeV}$. A neutrino telescope may measure both the GR-mediated $\bar{\nu}_{e}$ events $\left(N_{\bar{\nu}_{e}}^{\mathrm{GR}}\right)$ and the $\nu_{\mu}+\bar{\nu}_{\mu}$ events of charged-current (CC) interactions $\left(N_{\nu_{\mu}+\bar{\nu}_{\mu}}^{\mathrm{CC}}\right)$ in the vicinity of $E_{\bar{\nu}_{e}}^{\mathrm{GR}}$. Their ratio, defined as $R_{\mathrm{RG}} \equiv N_{\bar{\nu}_{e}}^{\mathrm{GR}} / N_{\nu_{\mu}+\bar{\nu}_{\mu}}^{\mathrm{CC}}$, can be related to the ratio of $\bar{\nu}_{e}$ 's to $\nu_{\mu}$ 's and $\bar{\nu}_{\mu}$ 's entering the detector,

$$
R_{0} \equiv \frac{\phi \bar{\nu}_{e}}{\phi_{\nu_{\mu}}^{\mathrm{D}}+\phi{\frac{\mathrm{D}}{\nu_{\mu}}}^{\mathrm{D}}} .
$$

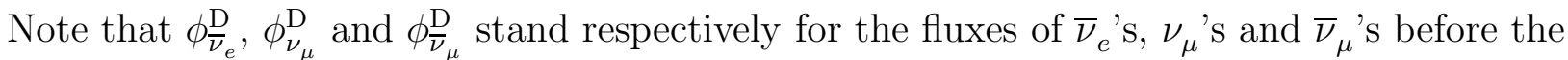
$\mathrm{RG}$ and $\mathrm{CC}$ interactions occur at the detector. In Ref. [13], $R_{\mathrm{GR}}=a R_{0}$ with $a \approx 30.5$ has been obtained by considering the muon events with contained vertices [14] in a water- or ice-based detector. An accurate calculation of $a$ is certainly crucial for a specific neutrino telescope to detect the GR reaction rate, but it is beyond the scope of this work. Instead, here we concentrate on the possible effect of $\mu-\tau$ symmetry breaking on $R_{0}$.

Provided the initial neutrino fluxes are produced via the decay of $\pi^{+}$'s and $\pi^{-}$'s created from high-energy $p p$ collisions, their flavor composition can be expressed in a more detailed way as follows:

$$
\left\{\phi_{\nu_{e}}, \phi_{\bar{\nu}_{e}}, \phi_{\nu_{\mu}}, \phi_{\bar{\nu}_{\mu}}, \phi_{\nu_{\tau}}, \phi_{\bar{\nu}_{\tau}}\right\}=\left\{\frac{1}{6}, \frac{1}{6}, \frac{1}{3}, \frac{1}{3}, 0,0\right\} \phi_{0} .
$$

In comparison, the flavor content of ultrahigh-energy neutrino fluxes produced from $p \gamma$ collisions reads ${ }^{1}$

$$
\left\{\phi_{\nu_{e}}, \phi_{\bar{\nu}_{e}}, \phi_{\nu_{\mu}}, \phi_{\bar{\nu}_{\mu}}, \phi_{\nu_{\tau}}, \phi_{\bar{\nu}_{\tau}}\right\}=\left\{\frac{1}{3}, 0, \frac{1}{3}, \frac{1}{3}, 0,0\right\} \phi_{0} .
$$

For either Eq. (20) or Eq. (21), the sum of $\phi_{\nu_{\alpha}}$ and $\phi_{\bar{\nu}_{\alpha}}$ (for $\left.\alpha=e, \mu, \tau\right)$ is consistent with $\phi_{\alpha}$ in Eq. (3).

\footnotetext{
${ }^{1}$ Note that the dominant reaction to generate electron and muon neutrinos in $p \gamma$ collisions is $p \gamma \rightarrow \Delta^{+} \rightarrow \pi^{+} n$ with $\pi^{+} \rightarrow \mu^{+} \nu_{\mu}$ and $\mu^{+} \rightarrow e^{+} \nu_{e} \bar{\nu}_{\mu}$. There is no production of $\bar{\nu}_{e}$, because the produced neutrons can escape the source before decaying [15]. In contrast, the numbers of $\nu_{e}$ 's and $\bar{\nu}_{e}$ 's in Eq. (20) are identical as a result of the equal amount of $\pi^{+}$'s and $\pi^{-}$'s produced from inelastic $p p$ collisions.
} 
Due to neutrino oscillations, the $\bar{\nu}_{e}$ flux at the detector of a neutrino telescope is given by $\phi \frac{\mathrm{D}}{\bar{\nu}_{e}}=\phi_{\bar{\nu}_{e}} \bar{P}_{e e}+\phi_{\bar{\nu}_{\mu}} \bar{P}_{\mu e}$. With the help of Eqs. (6), (10), (20) and (21), we explicitly obtain

$$
\begin{aligned}
\phi_{\bar{\nu}_{e}}^{\mathrm{D}}(p p) & =\frac{\phi_{0}}{6}(1-2 \Delta), \\
\phi_{\bar{\nu}_{e}}(p \gamma) & =\frac{\phi_{0}}{12}\left(\sin ^{2} 2 \theta_{12}-4 \Delta\right) .
\end{aligned}
$$

The sum of $\phi_{\nu_{\mu}}^{\mathrm{D}}$ and $\phi_{\bar{\nu}_{\mu}}^{\mathrm{D}}$, which is defined as $\phi_{\mu}^{\mathrm{D}}$, has been given in Eq. (12). It is then straightforward to calculate $R_{0}$ by using Eq. (19) for two different astrophysical sources:

$$
\begin{aligned}
& R_{0}(p p) \approx \frac{1}{2}-\frac{3}{2} \Delta, \\
& R_{0}(p \gamma) \approx \frac{\sin ^{2} 2 \theta_{12}}{4}-\frac{4+\sin ^{2} 2 \theta_{12}}{4} \Delta .
\end{aligned}
$$

This result indicates that the dependence of $R_{0}(p p)$ on $\theta_{12}$ is hidden in $\delta$ and suppressed by the smallness of $\epsilon$ and $\varepsilon$. In addition, the deviation of $R_{0}(p p)$ from $1 / 2$ can be as large as $1.5 \Delta_{\text {bound }} \approx 0.15$. It is obvious that the ratio $R_{0}(p \gamma)$ is very sensitive to the value of $\sin ^{2} 2 \theta_{12}$. A measurement of $R_{0}(p \gamma)$ at IceCube and other second-generation neutrino telescopes may therefore probe the solar neutrino mixing angle $\theta_{12}[13]$. Indeed, the dominant production mechanism for ultrahigh-energy neutrinos at Active Galactic Nuclei (AGNs) and Gamma Ray Bursts (GRBs) is expected to be the $p \gamma$ process in a tenuous or radiation-dominated environment [16]. If this expectation is true, the observation of $R_{0}(p \gamma)$ may also provide us with useful information on the breaking of $\mu-\tau$ symmetry.

4 In summary, we have discussed why and how the second-generation neutrino telescopes can serve as a striking probe of broken $\mu-\tau$ symmetry. Based on the conventional mechanism for ultrahigh-energy neutrino production at a distant astrophysical source and the standard picture of neutrino oscillations, we have shown that the flavor composition of cosmic neutrino fluxes at a terrestrial detector may deviate from the naive expectation $\phi_{e}^{\mathrm{D}}: \phi_{\mu}^{\mathrm{D}}: \phi_{\tau}^{\mathrm{D}}=1: 1: 1$. Instead, $\phi_{e}^{\mathrm{D}}: \phi_{\mu}^{\mathrm{D}}: \phi_{\tau}^{\mathrm{D}}=(1-2 \Delta):(1+\Delta):(1+\Delta)$ holds, where $\Delta$ characterizes the effect of $\mu-\tau$ symmetry breaking. The latter is actually a reflection of $\theta_{13} \neq 0$ and $\theta_{23} \neq \pi / 4$ in the $3 \times 3$ neutrino mixing matrix. We have examined the sensitivity of $\Delta$ to the deviation of $\theta_{13}$ from zero and to the departure of $\theta_{23}$ from $\pi / 4$, and obtained $-0.1 \lesssim \Delta \lesssim+0.1$ from current neutrino oscillation data. We find that it is also possible to probe the breaking of $\mu-\tau$ symmetry by detecting the $\bar{\nu}_{e}$ flux of $E_{\bar{\nu}_{e}} \approx 6.3 \mathrm{PeV}$ via the Glashow resonance channel $\bar{\nu}_{e} e \rightarrow W^{-} \rightarrow$ anything.

This work, different from the previous ones (see, e.g., Refs. [11-13,17]) in studying how to determine or constrain one or two of three neutrino mixing angles and the Dirac CPviolating phase with neutrino telescopes, reveals the combined effect of $\theta_{13} \neq 0, \theta_{23} \neq \pi / 4$ and $\delta \neq \pi / 2$ which can show up at the detector. Even if $\Delta \neq 0$ is established from the measurement of ultrahigh-energy neutrino fluxes, the understanding of this $\mu-\tau$ symmetry breaking signal requires more precise information about $\theta_{13}, \theta_{23}$ and $\delta$. Hence it makes sense to look at the complementary roles played by neutrino telescopes and terrestrial neutrino 
oscillation experiments (e.g., the reactor experiments to pin down $\theta_{13}$ and the neutrino factories or superbeam facilities to measure $\delta$ ) in the era of precision measurements.

The feasibility of our idea depends on the assumption that we have correctly understood the production mechanism of cosmic neutrinos from a distant astrophysical source (i.e., via $p p$ and $p \gamma$ collisions) with little uncertainties. It is also dependent upon the assumption that the error bars associated with the measurement of relevant neutrino fluxes or their ratios are much smaller than $\Delta$. The latter is certainly a challenge to the sensitivity or precision of IceCube and other neutrino telescopes under construction or under consideration, unless the effect of $\mu-\tau$ symmetry breaking is unexpectedly large. Nevertheless, there is no doubt that any constraint on $\Delta$ to be obtained from neutrino telescopes will be greatly useful in diagnosing the astrophysical sources and in understanding the properties of neutrinos themselves. Much more efforts are therefore needed to make in this direction.

The author would like to thank J.X. Lu for warm hospitality at the Interdisciplinary Center for Theoretical Study of USTC, where part of this paper was written. He is also grateful to H.B. Yu and S. Zhou for useful discussions. This work is supported by the National Natural Science Foundation of China. 


\section{REFERENCES}

[1] SNO Collaboration, Q.R. Ahmad et al., Phys. Rev. Lett. 89, 011301 (2002).

[2] For a review, see: C.K. Jung et al., Ann. Rev. Nucl. Part. Sci. 51, 451 (2001).

[3] KamLAND Collaboration, K. Eguchi et al., Phys. Rev. Lett. 90, 021802 (2003).

[4] K2K Collaboration, M.H. Ahn et al., Phys. Rev. Lett. 90, 041801 (2003).

[5] Particle Data Group, S. Eidelman et al., Phys. Lett. B 592, 1 (2004); Z.Z. Xing, Int. J. Mod. Phys. A 19, 1 (2004).

[6] A. Strumia and F. Vissani, Nucl. Phys. B 726, 294 (2005).

[7] See, e.g., T. Fukuyama and H. Nishiura, hep-ph/9702253; R.N. Mohapatra and S. Nussinov, Phys. Rev. D 60, 013002 (1999); Z.Z. Xing, Phys. Rev. D 61, 057301 (2000); Phys. Rev. D 64, 093013 (2001); E. Ma and M. Raidal, Phys. Rev. Lett. 87, 011802 (2001); C.S. Lam, Phys. Lett. B 507, 214 (2001); T. Kitabayashi and M. Yasue, Phys. Rev. D 67, 015006 (2003); W. Grimus and L. Lavoura, Phys. Lett. B 572, 189 (2003); J. Phys. G 30, 73 (2004); Y. Koide, Phys. Rev. D 69, 093001 (2004); R.N. Mohapatra, JHEP 0410, 027 (2004); A. de Gouvea, Phys. Rev. D 69, 093007 (2004); W. Grimus, A.S. Joshipura, S. Kaneko, L. Lavoura, H. Sawanaka, and M. Tanimoto, Nucl. Phys. B 713, 151 (2005); R.N. Mohapatra and W. Rodejohann, Phys. Rev. D 72, 053001 (2005); T. Kitabayashi and M. Yasue, Phys. Lett. B 621, 133 (2005); R.N. Mohapatra and S. Nasri, Phys. Rev. D 71, 033001 (2005); R.N. Mohapatra, S. Nasri, and H.B. Yu, Phys. Lett. B 615, 231 (2005); A.S. Joshipura, hep-ph/0512252; K. Matsuda and H. Nishiura, Phys. Rev. D 73, 013008 (2006); R.N. Mohapatra, S. Nasri, and H.B. Yu, Phys. Lett. B 636, 114 (2006); hep-ph/0605020.

[8] J. Ahrens et al. (IceCube), Nucl. Phys. (Proc. Suppl.) 118, 388 (2003).

[9] E. Aslanides et al. (ANTARES), astro-ph/9907432; S.E. Tzamarias (NESTOR), Nucl. Instrum. Meth. A 502, 150 (2003); P. Piattelli (NEMO), Nucl. Phys. (Proc. Suppl.) 143, 359 (2005). The web addresses of these three collaborations are http://antares.in2p3.fr/, http://www.nestor.org.gr/ and http://nemoweb.Ins.infn.it/, respectively.

[10] S.L. Glashow, Phys. Rev. 118, 316 (1960).

[11] W. Winter, hep-ph/0604191; and references therein.

[12] Z.Z. Xing and S. Zhou, astro-ph/0603781.

[13] P. Bhattacharjee and N. Gupta, hep-ph/0501191.

[14] J.F. Beacom, N.F. Bell, D. Hooper, S. Pakvasa, and T.J. Weiler, Phys. Rev. D 68, 093005 (2003); Erratum-ibid. D 72, 019901 (2005).

[15] M. Ahlers, L.A. Anchordoqui, H. Goldberg, F. Halzen, A. Ringwald, and T.J. Weiler, Phys. Rev. D 72, 023001 (2005); and references therein.

[16] V.S. Berezinsky, S.V. Bulanov, V.A. Dogiel, V.L. Ginzburg, and V.S. Ptuskin, Astrophysics of Cosmic Rays (North-Holland, Amsterdam, 1990); T.K. Gaisser, F. Halzen, and T. Stanev, Phys. Rept. 258, 173 (1995).

[17] G. Barenboim and C. Quigg, Phys. Rev. D 67, 073024 (2003); J.F. Beacom, N.F. Bell, D. Hooper, S. Pakvasa, and T.J. Weiler, Phys. Rev. D 69, 017303 (2004); P.D. Serpico and M. Kachelrieß, Phys. Rev. Lett. 94, 211102 (2005); P.D. Serpico, Phys. Rev. D 73, 047301 (2006). 


\section{FIGURES}
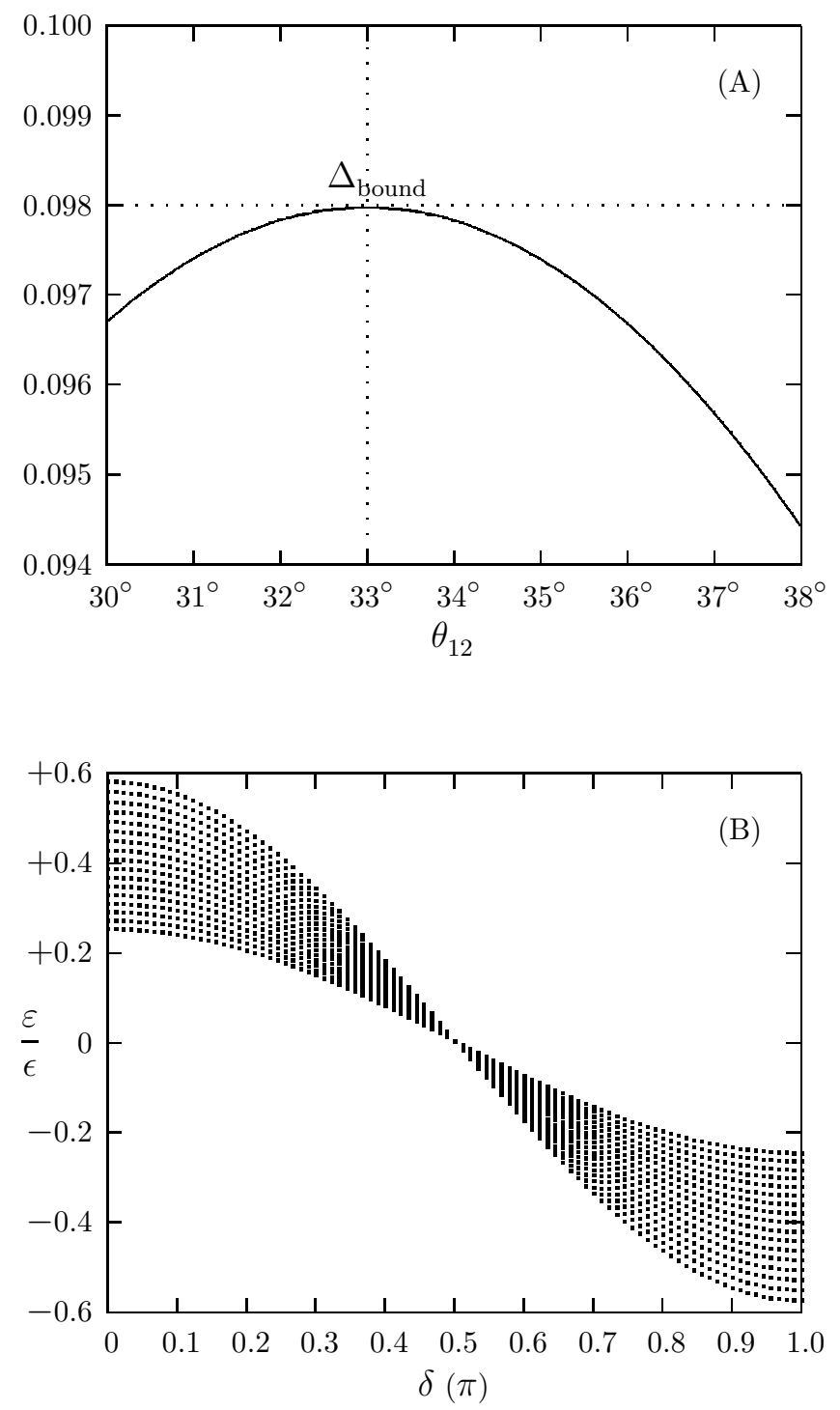

FIG. 1. (A) the dependence of $\Delta_{\text {bound }}$ on $\theta_{12}$, where $\epsilon$ and $\varepsilon$ take their respective upper limits (i.e., $\epsilon<10^{\circ}$ and $\varepsilon<9^{\circ}$ ); (B) the nontrivial condition for $\Delta=0$, where $\theta_{12}$ is allowed to vary in the range $30^{\circ}<\theta_{12}<38^{\circ}$. 